\title{
ASPECTOS EPISTEMOLÓGICOS EM ESTUDOS SOBRE ANÁLISE DE DOMÍNIO SOB AS LENTES DO RACIONALISMO E DO EMPIRISMO
}

\author{
Igor Soares Amorim ${ }^{1}$ \\ Universidade Federal de Santa Catarina - UFSC \\ igao.sa@gmail.com \\ William Barbosa Vianna ${ }^{2}$ \\ Universidade Federal de Santa Catarina \\ william.vianna@ufsc.br \\ Marisa Brasher Basilio Medeiros ${ }^{3}$ \\ Universidade Federal de Santa Catarina - UFSC \\ marisa.brascher@gmail.com
}

\begin{abstract}
Resumo
Objetiva identificar e analisar as categorias teórico-metodológicas em que se situam os estudos sobre Análise de Domínio publicados na Base Lisa ) Library and Information Science Abstracts) tendo em vista o desenvolvimento do assunto. Justifica-se pela relevância da incorporação de contribuições interdisciplinares a partir de marcos epistemológicos bem delimitados em vista da polissemia e idiossincrasia dos termo "análise" e "domínio. A busca recuperou 80 artigos, dos quais 50 demonstraram aderência ao estudo. Concluiu-se que embora a análise do domínio seja uma proposta característica do paradigma social da Ciência da Informação, sua aplicação preserva perfis mais tradicionais.
\end{abstract}

Palavras-chave: Epistemologia. Análise de Domínio. Metodologia. Empirismo. Racionalismo.

\section{EPISTEMOLOGICAL ASPECTS IN STUDIES ON DOMAIN ANALYSIS UNDER RATIONALISM AND EMPIRISM LENSES}

\begin{abstract}
It aims to identify and analyze the theoretical-methodological categories in which the studies on Domain Analysis published in the Lisa Base are located in order to develop the subject. It is justified by the relevance of the incorporation of interdisciplinary contributions from well-defined epistemological frameworks in view of the polysemy and idiosyncrasy of the term "analysis" and "domain. The search retrieved 80 articles, of which 50 showed adherence to the study. It was concluded that although the domain analysis is a characteristic proposal of the social paradigm of Information Science, its application preserves more traditional profiles.
\end{abstract}

Keywords: Epistemology. Domain Analysis. Methodology. Empiricism. Rationalism.

\footnotetext{
1 Formado em Ciências da Informação, Documentação e Biblioteconomia pela Universidade de São Paulo. Integrante do programa de pós-graduação em Ciência da Informação da Universidade Federal de Santa Catarina. É representante discente e membro do conselho fiscal da Associação Nacional de Pesquisa e Pós-Graduação em Ciência da Informação (Ancib).

2 Doutorado em Engenharia de Produção (2011). Mestrado Profissional em Administração (USF, 2004). Mestrado em Engenharia de Produção (UFSC, 2008). Graduação em Ciências Humanas - Filosofia (UCDB1989).

${ }^{3}$ Professora do Departamento de Ciência da Informação da Universidade Federal de Santa Catarina. Mestrado e doutorado em Ciência da Informação pela Universidade de Brasília, com dois anos de pesquisa na Université de Nice Sophia Antipolis (França).
}

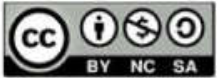




\section{INTRODUÇÃO}

A Análise de Domínio (AD) tem se consolidado a partir da década de 1990 como uma das mais relevantes disciplinas para organização da informação em contextos específicos de comunidades de discurso e de práticas no campo da Biblioteconomia e da Ciência da Informação (BCI).

Em 1995, a análise de domínio foi definida e desenvolvida na área principalmente pelos trabalhos de Birger Hjørland e Albrechtsen (HJØRLAND; ALBRECHTSEN, 1995) e posteriormente por outros, tanto sobre a teoria quanto sobre a aplicação dos princípios em contextos específicos.

Guimarães (2014), corrobora que a análise de domínio vem sendo tradicionalmente trabalhada em termos teóricos e aplicados, por Hjørland e Albrechtsen (1995) e ainda MoyaAnegón \& Herrero-Solana (2001), Hjørland (2002a, 2002b, 2008), Tennis (2003), e Smiraglia (2011), dentre outros (GUIMARÃES, 2014), constituindo-se em marcante abordagem para caracterização e avaliação da ciência, na medida em que permite identificar as condições nas quais o conhecimento científico se constrói e se socializa, caracterizando-se, dessa forma, como relevante contribuição para Ciência da Informação.

Nesse sentido, considera-se que os estudos sobre análise de domínio além de relevantes, demandam no atual estagio da sociedade da informação, pela sua natureza aplicada, interações interdisciplinares, tendo em vista seu desenvolvimento em diversificados contextos sociais em sua rápida expansão de ambientes físicos para ambientes digitais e mistos, dentre outros desafios.

Entretanto, a literatura dá conta que não obstante as tentativas de alguns autores em conceituar o que é domínio, são identificadas constantes críticas e questionamentos quanto à falta de explicação do que constitui um domínio e de como o mesmo é formado (PALMER, 1999; TALJA; TUOMINEN; SAVOLAINEN, 2005).

O enfrentamento desse desafio epistemológico é fundamental para o desenvolvimento teórico e prático da $\mathrm{AD}$, ou seja, uma nova atitude frente à questão do conhecimento, no caso, em lócus bem delimitados com possibilidade de contribuições de diversas disciplinas científicas além das tradicionais.

Para tanto, Hjorland (2005) indica que a melhor forma de conhecer um domínio é identificar quais são as teorias atuantes nos mesmos: teorias ontológica, epistemológica e 
sociológica.

E Lakatos (1987) completa que ao se fomentar uma abertura dos clusters teóricos para mudar ou aperfeiçoar os pressupostos frente a novas realidades teóricas e empíricas é necessária uma filosofia da ciência situada (LAKATOS, 1979, p.11). E ainda que a filosofia da ciência sem a história da ciência é vazia; a história da ciência sem a filosofia da ciência é cega (LAKATOS, 1983, p. 107).

Nessa perspectiva, considera-se que identificar os clusters teóricos e práticas metodológicas associadas e as afiliações epistemológicas pode facilitar a tarefa de identificar possibilidades de desenvolvimento interdisciplinar de forma disciplinada.

Dessa forma, pergunta-se: como se categorizam as principais abordagens de análise de domínio do ponto de vista teórico-metodológico?

O objetivo do estudo é identificar a partir dos métodos utilizados, como se situam os estudos de $\mathrm{AD}$ frente aos paradigmas racionalista e empirista quanto à frequência de ocorrências tendo em vista identificar oportunidades de desenvolvimento interdisciplinar do tema.

Para alcançar o objetivo, serão percorridas as seguintes etapas:

a) Levantamento em base de dados internacionais de artigos que tratem especificamente da análise de domínio;

b) Leitura e exame de tais artigos, buscando categorizar a manifestação da análise de domínio em categorias teóricas e metodológicas.

A principal justificativa do estudo é que a diversidade de contextos em que se aplica a $\mathrm{AD}$ pode demandar aporte de conhecimentos advindos de outras áreas de conhecimento o que exige disciplina intelectual e que os estudos de $\mathrm{AD}$ sejam bem caracterizados e situados epistemologicamente para que se avance de forma consistente no diálogo interdisciplinar.

A análise se dará a partir de duas categorias propostas por Hjørland (2002b), consideradas "lentes epistemológicas" (racionalismo e empirismo) para compreensão de um domínio, uma vez que representam uma simplificação de escolas epistemológicas de maior amplitude.

A primeira etapa deste trabalho consistiu na identificação de características fundantes do racionalismo e do empirismo com base nas acepções de Descartes e Hume. Em seguida, definiu-se a base de dados Library and Information Science Abstracts - LISA, da Wilson Library Literature and Information Science Full Text. A Lisa foi selecionada por ser uma base de resumos de artigos voltada à área da $\mathrm{BCI}$ e, atualmente, cobre mais de 440 periódicos de 45 países. 
Utilizou-se o termo “domain analysis" como estratégia de busca, mantido entre aspas. Filtramos os resultados por dois critérios: língua em inglesa e período entre "1 de janeiro de 1990 à 31 de agosto de 2016", especificando o idioma em inglês, espanhol e português e o tipo de fonte em "periódicos acadêmicos".

$\mathrm{Da}$ analise emergiram as afiliações teóricas que aproximaram a produção do racionalismo, empirismo ou outros, bem como identificou os principais métodos utilizados.

\section{AS MATRIZES EPISTEMOLÓGICAS DO RACIONALISMO E DO EMPIRISMO E OS DESAFIOS PARA OS ESTUDOS SOBRE ANÁLISE DE DOMÍNIO}

Segundo Moser, Mulder e Trout (2008, p. 6), epistemologia é o estudo filosófico da natureza, das fontes e dos limites do conhecimento. A epistemologia é uma disciplina normativa que visa distinguir o conhecimento verdadeiro do conhecimento ilusório e empreende esta tarefa para melhorar os meios pelos quais as pessoas podem adquirir conhecimentos cientificamente válidos, levando-se em conta o conhecedor e o conhecido.

Tradicionalmente a epistemologia formulou diversos postulados sobre as fontes do conhecimento, das quais podemos pontuá-las genericamente sobre dois polos opositores: o polo racionalista (que enfoca os conhecimentos ditos inatos e universais) e o polo empirista (enfoca os fenômenos observáveis). Cada um dos polos tem uma perspectiva própria da relação conhecedor-conhecido-conhecimento.

Embora para alguns filósofos a epistemologia seja um campo mais amplo e que engloba (ou que deveria englobar) todos os domínios científicos, para outros é possível desenvolver uma epistemologia restrita a um único domínio a fim de definir sua categoria de conhecimento específica.

Essa perspectiva se apoia principalmente nos efeitos causados pelas contribuições de Wittgenstein que nega a "existência de uma única noção geral de conhecimento que esteja por trás de vários domínios epistêmicos potenciais" (MOSER; MULDER; TROUT, 2008, p. 30).

Num sentido aplicado à BCI, Hjørland e Albrechetsen (1995) consideram que as perspectivas sociológicas e filosóficas são fundamentais para os estudos da informação em domínios. A análise de domínio não centra sua atenção sobre a informação ou no conhecimento, mas antes, na produção destes. Assim, seu foco é sobre as comunidades discursivas, as quais são compostas por saberes, práticas, objetos, sujeitos e instituições sociais em constante interação tecem redes de signos e semânticas articulados em documentos. 
Hjørland e Albrechtsen (1995) apontam para carência de abordagens epsitemologicas em $\mathrm{AD}$ que não as tradicionais. Segundo Hjørland (2008) há urgência em aplicar diversificados postulados epistemológicos em domínios, sobretudo das ciências humanas e sociais, sendo que há poucos trabalhos nesse sentido.

Os autores se referem a domínio, apenas como "comunidades de pensamento ou comunidades discursivas que integram a divisão social do trabalho" (HJØRLAND; ALBRECHTSEN, 1995, p. 401).

Dessa forma, embora a proposta seja uma das mais relevantes, categorizar as matrizes teórico-práticas daquilo que se tem produzido sobre o tema pode lançar luzes sobre os marcos conceituais e metodológicos utilizados e evidenciar tendências e perspectivas para o desenvolvimento do tema, destacando-se que não existe uma definição exata e consensual sobre o quê realmente seria a "análise de domínio". Alem disso, o conceito é polissêmico e idiossincrático, composto de dois termos o primeiro que remete a ação, "analisar", e o segundo remete ao objeto que recebe tal ação, o "domínio".

A "análise" pode ser compreendida como análise textual, terminológica, estatística, estudos de comunidades, de documentos, de instituições. E o "domínio" pode ser uma disciplina, um grupo de pessoas, uma instituição, uma grande área do saber, um ramo muito específico do saber, ou até mesmo uma empresa.

Em vista de categorizar a produção intelectual, têm-se que, segundo a perspectiva racionalista, a principal fonte de conhecimento é a razão, enquanto para os empiristas, a maior fonte de conhecimento é a experiência sensorial.

Destacam-se entre os principais representantes de cada perspectiva Descarte (1983) e Hume (1972), respectivamente, sendo que, é sob as acepções básicas desses dois autores que se assenta a análise desenvolvida no presente estudo, considerando-as centrais para compreensão e organização dos diversos estudos sobre AD.

\subsection{O RACIONALISMO EM DESCARTES}

Em busca do conhecimento verdadeiro, Descartes (1983, p. 85), parte da consideração de que todo conhecimento que não seja "inteiramente certo e indubitável" deve ser considerado ilusório.

Segundo o filósofo, diversos conhecimentos são resultados de enganos. Como não temos condições de saber realmente se os conhecimentos adquiridos pelos sentidos são verdadeiros, posto que tudo que sentimos pode ser sentido não apenas na realidade, como 
também em sonho, todo conhecimento sensível é tomado como ilusão.

O que seria verdade, então? Para Descartes (1983, p. 87) há elementos muito simples e muito gerais que contém alguma coisa de verídico: "Pois, quer eu esteja acordado, quer esteja dormindo, dois mais três formarão sempre o cinco e o quadrado nunca terá mais do que quatro lados; e não parece possível que verdades tão patentes possam ser suspeitas de alguma falsidade ou incerteza".

A suspeita sobre os sentidos recai até mesmo sobre o corpo, do qual temos ciência senão pelos sentidos. Contudo, na mesma medida em que é possível duvidar do corpo, não é possível duvidar de alguém que duvide.

Isto implica, segundo Descartes (1983, p. 92), num “eu" existente e independente do corpo ou de sentidos, um eu que duvida, que pensa a dúvida, "ter por constante que a proposição, eu sou, eu existo, é necessariamente verdadeira todas as vezes que a enuncio ou que a concebo em meu espírito".

Procurando um atributo inseparável desse eu, Descartes (1983, p. 93-94), encontra o "pensar": "o pensamento é um atributo que me pertence; só ele não pode ser separado de mim. Eu sou, eu existo: isto é certo; mas por quanto tempo? A saber, por todo tempo em que eu penso. [...] nada sou, pois, falando precisamente, senão uma coisa que pensa". O pensamento como única via de verdade submete as sensações à ele próprio (submete a imaginação ao entendimento).

As coisas externas a meu pensamento, o mundo material só existiria assim em minha imaginação. Contudo, Descartes acredita que em tudo o quê nos é informado da natureza (do mundo externo ao pensamento), há algo de verdadeiro, e que temos condições para alcançar essa verdade. A natureza nos ensina, segundo exemplos do filósofo, que há uma contiguidade entre corpo e pensamento, que há outros corpos que interagem com o meu. Contudo todos esses ensinamentos ao espírito são "confusos e obscuros" (DESCARTES, 1983, p. 138).

E ponto de vista das implicações metodológicas do racionalismo baseado no pensamento cartesiano temos quatros regras: a) jamais aceitar algo como verdadeiro enquanto não fosse verificado (regra da evidencia), b) é preciso dividir em pequenas partes as dificuldades para examiná-las mais de perto (regra da análise), c) é preciso impor uma ordem aos pensamentos (regra da síntese) e, d) fazer enumerações e revisões para não correr o risco de omissões (regra da enumeração). 


\subsection{O EMPIRISMO EM HUME}

Com relação ao empirismo, a crença é de que o conhecimento advém da experiência. Segundo o inglês David Hume o pensamento mais vivo é sempre inferior à sensação mais embaçada (HUME, 1972, p. 16).

A frase de Hume (1972), marca a distância de seu olhar com o de Descartes (1983), dotando a sensação como fundamento para o conhecimento, e não alguma habilidade inata, embora o pensamento possa, por meio da imaginação ou da memória, trazer alguma ideia imprecisa ao entendimento. Mais forte que essas ideias são as impressões, resultado de "todas as nossas percepções mais vivas, quando ouvimos, vemos, sentimos, amamos, odiamos, desejamos ou queremos" (HUME, 1972, p. 16). Dessa forma, as ideias são consideradas cópias das impressões, cabendo ao filósofo se questionar qual é a impressão original de qualquer ideia.

Se Descarte (1983), duvidava dos sentidos e creditava a verdade ao pensamento, Hume dirá o contrário, que a verdade está na internalização da natureza por meio dos sentidos, e que ao pensamento cabe à constituição de cópias das impressões e a criação de conexões distintas entre estas cópias. Tais conexões permite o encadeamento das ideias. Para Hume (1972, p. 21) há três princípios básicos de conexão entre as ideias, o de semelhança, o de contiguidade (mesmo espaço/tempo) e o de causa-efeito.

Quando voltamos à questão das relações aos fatos, ao mundo cotidiano, os raciocínios fundam-se na relação causa-efeito, o que lava-nos a constante suposição de que "há uma conexão entre o fato presente e aquêle que é inferido dêle" (HUME, 1972, p. 31). Dessa forma, investigar o conhecimento verdadeiro incidiria no entendimento de como compreendemos a relação de causa e efeito.

Hume (1972) afirma que esse conhecimento deriva da experiência, e não necessariamente da razão, pois o efeito é de natureza distinta da causa, o que denota a arbitrariedade presente na relação causa-efeito: "todos os raciocínios abstratos do mundo não poderão jamais nos levar a dar um passo para chegar a conhece-la” (HUME, 1972, p. 35).

\section{RESULTADOS E DISCUSSÕES}

Na busca na base de dados Library and Information Science Abstracts - LISA, da Wilson Library Literature and Information Science Full Text, com o termo "domain analysis" foram obtidos 80 artigos. Um desses não era uma um artigo de periódico, mas um "Feature" e outro era uma conferência. Restaram, portanto, 78 publicações como artigos de periódicos. 
Com a primeira filtragem identificou-se 18 artigos de abordagens exclusivamente teóricas e que foram descartados por não fazerem parte do escopo de interesse desta pesquisa que é a identificação de afiliações teórico-metodológicas.

Da análise dos 60 restantes, em 9 artigos os resumos foram insuficientes para identificar se o estudo era de natureza aplicada ou teórica. Nesse grupo, foram então analisados também os títulos, as palavras-chave e uma leitura do texto focada, buscando identificar a metodologia utilizada na aplicação da análise de domínio, sendo que 3 artigos foram considerados teóricos e um não foi encontrado, impossibilitando uma a leitura e sua análise, chegando-se a 56 . De uma nova leitura completa, resultou o descarte de mais 6 artigos que ou eram teóricos ou que não relacionados à análise de domínio.

Por fim, a amostra foi composta por 50 artigos, todos trazendo uma aplicação da análise de domínio, como mote central do texto, ou como uma exemplificação de uma proposta em questão das pesquisas.

A categorização em racionalista ou empirista se deu com base na auto-declaração realizada pelos autores em sua definição de natureza e/ou com base nas características apresentadas nos tópicos anteriores sobre Descartes (1983) e Hume (1972). Dessa forma, apresenta-se conforme o quadro abaixo.

Quadro 1: Epistemologias nas análises de domínio

\begin{tabular}{|l|r|}
\hline Epistemologias & \multicolumn{1}{|c|}{ Qtd. } \\
\hline Empirista & $\mathbf{2 9}$ \\
\hline Racionalista & $\mathbf{1 3}$ \\
\hline Outros & $\mathbf{8}$ \\
\hline $\mathrm{N}^{\circ}$ de artigos & 50 \\
\hline
\end{tabular}

Fonte: elaborado pelos autores

Conforme o quadro Y, a epistemologia Empirista, relacionada ao paradigma físico da Ciência da Informação, é a abordagem mais frequente nos artigos que aplicam análise de domínio, constando em 29 artigos.

Em seguida, a abordagem Racionalista aparece em 13 artigos. Por fim, outras abordagens são no total de 8 , sendo que ma análise mais profunda seria necessária para identificar a afiliação desses 8 artigos em relação às categorias propostas e/ou outro enquadramento teórico.

Com base nesses dados, verifica-se que uma predominância de afiliações teóricas aos modelos racionalistas e empiristas, quando se poderia esperar uma produção maior em outros, por exemplo, como representação de outros modelos epistemológicos. No gráfico a seguir é 
possível identificar a distribuição de forma percentual.

Gráfico 1: Distribuição de forma percentual

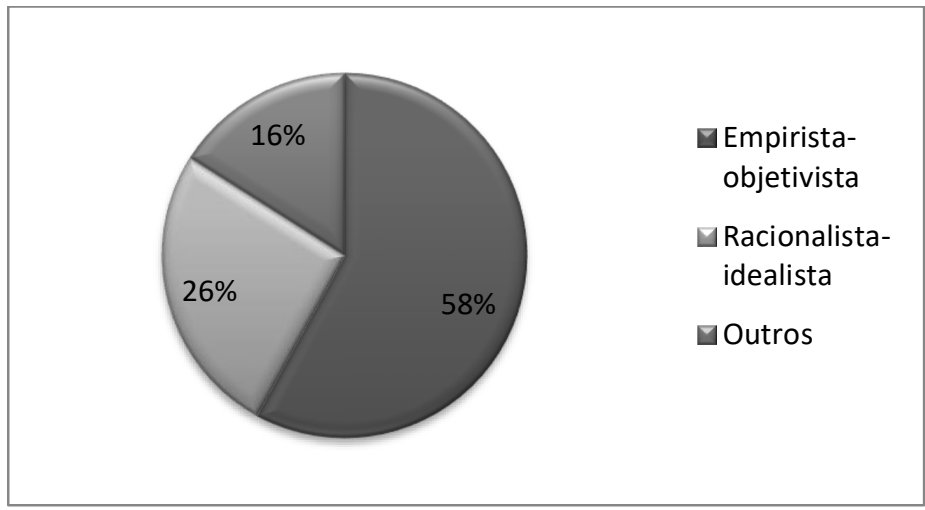

Fonte:

$\mathrm{Na}$ sequencia da análise, foram identificadas as metodologias utilizadas nos artigos, conforme a afiliação. O grupo relativo a epistemologia Racionalista apresentou uma distribuição mais equilibrada entre 3 métodos gerais. Destacou-se a categorização e a modelização (aplicação de um grupo de classes, categorias, modelos ou facetas num movimento dedutivo), respectivamente com 6 e com 5 aparições, e com menos destaque, a mineração textual, com 2 (quadro 2):

Quadro 2:

\begin{tabular}{|l|r|}
\hline \multicolumn{1}{|c|}{ Racionalista-idealista } & \multicolumn{2}{c|}{ Resultados } \\
\hline Categorização & 6 \\
\hline Modelização Computacional & 5 \\
\hline Mineração Textual & 2 \\
\hline Total & $\mathbf{1 3}$ \\
\hline
\end{tabular}

Fonte: elaborado pelos autores.

No grupo de artigos designados como empiristas, a grande maioria dos estudos utilizaram-se das métricas como instrumento de análise, com apenas dois trabalhos fugindo à regra, um utilizando a pesquisa documental (WHITE, 2001), e outro o questionário para o estudo de usuário (FREUND, 2013) (quadro 3):

Quadro 3:

\begin{tabular}{|l|r|}
\hline \multicolumn{1}{|c|}{ Empirista } & \multicolumn{2}{c|}{ Resultados } \\
\hline Pesquisa Documental & 1 \\
\hline Questionário & 27 \\
\hline Estudos métricos & $\mathbf{2 9}$ \\
\hline Total & \\
\hline
\end{tabular}

Fonte: elaborado pelos autores. 
Os resultados demonstram uma hegemonia da aplicação métrica, seguida da categorização e modelização.

Hjørland (2002a, 2002b, 2005), afirma a necessidade da BCI se abrir a novas perspectivas teóricas e práticas, além das baseadas no racionalismo e no empirismo. Quando voltamos à discussão para a análise de domínio, uma abordagem teórico-metodológica voltada para o social, que dialoga com as Ciências Sociais, Humanidades e Filosofia, por exemplo, espere-se que em suas aplicações encontremos uma fundamentação teórica desse tipo. Contudo, não foi o que constatamos na análise dos dados até aqui.

Entretanto, na categoria "Outros", embora o número de trabalhos seja menor do ponto de vista estatístico de representatividade, pouco mais de $10 \%$, foram encontrados maior variedade de métodos, (quadro 4):

Quadro 4:

\begin{tabular}{|l|r|}
\hline \multicolumn{1}{|c|}{ Outros } & \multicolumn{1}{c|}{ Qtd. } \\
\hline Análise Fenomenológica & 2 \\
\hline Etnografia & 2 \\
\hline Cognitive Work Analisys & 2 \\
\hline Análise Histórica & 1 \\
\hline Análise do discurso & 1 \\
\hline Total & $\mathbf{8}$ \\
\hline
\end{tabular}

Fonte: elaborado pelos autores.

Verifica-se no grupo definido como "outros" houve uma maior variedade de metodologias que são comumente utilizadas em estudos qualitativos em ciências sociais e tem por objetivo traduzir e expressar o sentido dos fenômenos do mundo social; tratando-se de reduzir a distância entre indicador e indicado, entre teoria e dados, entre contexto e ação (MAANEN, 1979, p. 520).

\section{CONSIDERAÇÕES FINAIS}

De maneira geral, os resultados da busca com o uso do têrmo "domain analysis", na base LISA permitiu uma categorização teórico-metodológica baseada nas perspectivas racionalista e empírica que pode contribuir com o desenvolvimento do tema em BCI a partir de algumas constatações e lacunas.

Primeiramente, verificou-se que os estudos predominantemente se situam na matriz empirista (29) e racionalista (13). Maior interesse despertam os estudos categorizados como outros (8), pelo potencial de contribuição com abordagens emergentes, considerando-se que a 
base de dados é considerada altamente qualificada na área, ou seja, está fundamentada numa rigorosa revisão por pares, o que legitima sua produção.

Em segundo lugar, destacam-se os métodos utilizados, sendo que particularmente chama a atenção a associação dos estudos métricos com a matriz empirista, quando tradicionalmente nas ciências, tais métodos estão associados ao racionalismo, o que merece maior aprofundamento. Em geral, o racionalismo se baseia na matemática e o empirismo nas ciências naturais e na observação direta de experiências repetidas, além do fato de que nesta vertente, a indução substitui a dedução.

Por outro lado, os métodos utilizados em "outros", aproximam-se mais daqueles utilizados em estudos qualitativos, o que pode ser uma oportunidade para o aprofundamento dos estudos de $\mathrm{AD}$, considerando-se o forte envolvimento das particularidades do contexto social de comunidades discursivas nesse tipo de estudo.

Embora, discursivamente, não raro na pesquisa na BCI as propostas de análise de domínio sejam colocadas no escopo do paradigma social, teórico- metodologicamente não se constatou isso no fragmento da literatura analisado, ao contrário.

As abordagens epistemológicas da Análise de Domínio que utilizam de métodos predominantemente empíricos ou racionalistas podem não condizer com uma concepção do paradigma social. Tais abordagens, de um ponto de vista epistemológico, parecem reforçar os paradigmas fisicistas e cognitivistas da BCI. A análise de domínio, sendo proposta por Hjørland (1995), na década de 1990 objetivava adentrar a novas perspectivas, tais como a hermenêutica, a teoria crítica, o pragmatismo, o pós-estruturalismo, dentre outras. Assim, notamos um descompaço entre as aplicações e o espaço epistemológico da proposta hjørlandiana.

Uma vez constatado um baixo índice de produção sob a epistemologia do paradigma "social", entende-se como uma carência da área que justificaria pesquisas e aprofundamentos nesse sentido, tanto na produção identificada como "outros", quanto em outras bases de dados com outro têrmos de busca, tendo em vista maiores aprofundamentos do assunto. 


\section{REFERENCIAS}

BROOKES, B. C. The foundations of information science: part I. Philosophical aspects. Journal of Information Science, v. 2, p. 125- 133,1980.

DESCARTES, R. Coleção Os Pensadores. São Paulo: Abril Cultural, 1983.

DUARTE, A. B. S. Ciclo informacional: a informação e o processo de comunicação. Em Questão, v. 15, n. 1, p. 57-72, 2009.

GUEDES, W.; ARAÚJO JÚNIOR, R. H. O fluxo de informações na auditoria pública e a teoria Matemática da Comunicação. Encontros Bibli: revista eletrônica de biblioteconomia e ciência da informação, v. 19, n. 40, p. 33-50, 2014.

GUIMARÃES, J. A. C. Análise de domínio como perspectiva metodológica em organização da informação. Ciência da Informação, v. 41, n. 1, p.13-21, jan./abr., 2014.

HJØRLAND, B. Comments on the articles and proposals for further work. Journal of documentation, v. 61, n. 1, p. 156-163, 2005.

HJØRLAND, B.; ALBRECHTSEN, H. Toward a New Horizon in Information Science:

Domain-Analysis. JASIS, v. 46, n. 6, p. 400-425, 1995.

Domain analysis in information science: Eleven approaches-traditional as well as

innovative. Journal of Documentation, v. 58, n. 4, p. 422-462, 2002a.

Epistemology and the socio_cognitive perspective in information science. Journal of the American Society for Information Science and Technology, v. 53, n. 4, p. 257-270, 2002b.

. Deliberate bias in knowledge organization? In: INTERNATIONAL ISKO CONFERENCE, 10., 2008, Montréal, Proceedings... Montréal: ISKO, 2008. p. 256-261.

HUME, D. Investigação acerca do entendimento humano. São Paulo: Nacional/EDUSP, 1972.

LAKATOS, I. O falseamento e a metodologia dos programas de pesquisa científica. In: LAKATOS, I.; MUSGRAVE, A. (Org.). A crítica e o desenvolvimento do conhecimento. São Pao: Cultrix, 1979.

Historia de las ciencias y sus reconstrucciones racionales. Madrid: Tecnos, 1987.

LE COADIC, Y. F. A Ciência da Informação. 2. ed. Trad. de Maria Yêda F. S. F. G. Brasília: Briquet de Lemos Livros, 2004.

A matemática da informação. In: TOUTAIN, L. M. B. B. Para entender a ciência da informação. Salvador: EDUFBA, 2007. p. 219-239.

MAANEN, J. V. Reclaiming qualitative methods for organizational research: a preface. Administrative Science Quarterly, v. 24, n. 4, p 520-526, December 1979. 
MOYA y ANEGÓN, F.; HERRERO SOLANAS, V. Análisis de domino de la revista mexicana Investigación Bibliotecológica. Información, Cultura y Sociedad, v. 5, p. 10-28, 2001 .

MOSER, P. K.; MULDER, D. H.; TROUT, J. D. A teoria do conhecimento: uma introdução temática. 2. ed. São Paulo: WMF Martins Fontes, 2008.

PIMENTA, S. G. Conceitos de informação e texto nas abordagens do ponto de vista cognitivo na Ciência da Informação e do processamento da informação na psicologia cognitiva - uma visão interdisciplinar. Encontros Bibli: Revista Eletrônica de Biblioteconomia e Ciência da Informação, v. 17, n. esp.1, p. 40-66, 2012.

ROBREDO, J. Da ciência da informação revisitada aos sistemas humanos de informação. Brasília: Tesaurus, 2003.

SALDANHA, G. S. A grande bibliologia: notas epistemológico-históricas sobre a ciência da organização dos saberes. Transinformação, v. 28, n. 2, p.195-207, 2016.

SALDANHA, G. S. Tradições epistemológicas nos estudos de organização dos saberes: uma leitura histórico-epistêmica a partir da filosofia da linguagem. Liinc em Revista, v. 6, n. 2, p. 300-315, 2010.

SILVA, A. M. O método quadripolar e a pesquisa em ciência da informação. Revista Prisma, n. 26, p. 27-44, 2014.

TALJA, S.; TUOMINEN, K.; SAVOLAINEN, R. "Isms" in information science: constructivism, collectivism and constructionism. Journal of Documentation, v. 61, n. 1, p. 79-101, 2005. 